\title{
A Study on the Agricultural Reservoir Behavior through Numerical Analysis and the Centrifuge Model Test in Earthquakes
}

\author{
Joon Heo ${ }^{1}$, Taeho Bong' ${ }^{2}$, Sangok Jeon ${ }^{3}$, Yongseong Kim ${ }^{4}$ \\ ${ }^{1}$ Rural Research Institute/Korea Rural Community Corporation \\ E870, Haen-ro Sangnok-gu, Ansan-si, Republic of Korea \\ jheo01@ekr.or.kr \\ ${ }^{2}$ College of Engineering/Seoul National University \\ 1 Gwanak-ro Gwanak-gu, Seoul, Republic of Korea \\ bth21@snu.ac.kr \\ ${ }^{3}$ Rural Research Institute/Korea Rural Community Corporation \\ E870, Haen-ro Sangnok-gu, Ansan-si, Republic of Korea \\ jeonso@ekr.or.kr \\ ${ }^{4}$ Department of Regional Infrastructure Engineering/Kangwon National University \\ 1 Kangwondaehak-gil, Chuncheon-si, Republic of Korea \\ yskim2@kangwon.ac.kr
}

\section{Extended Abstract}

Most of the South Korean agricultural facilities are dilapidated due to old age. They are highly likely to be damaged in the event of natural disasters like extreme weather and earthquakes. If the facilities suffer defects, it may cause unexpected massive emergencies and damage to human life and property. If agricultural infrastructures lose their functions due to earthquakes, it may cause unexpected economic and industrial damage to the surrounding and downstream areas. As seen in the past similar cases, a local damage alone due to earthquakes may have considerable economic and industrial impacts. Thus, to define the vulnerability of agricultural infrastructures for ensuring seismic reinforcement, there is a need to evaluate the seismic performance of reservoir embankments according to the seismic wave characteristics (short cycle, long cycle, artificial seismic wave) [1-3].

The seismic performance evaluation of agricultural infrastructures was conducted using the time history analysis method of the three-dimensional dynamic analysis methods in analyzing the embankment displacement and its acceleration amplification characteristics in agricultural infrastructures according to the seismic wave characteristics. Based on the ground investigation data, the representative ground cross-section and the ground parameters were set. Ground response analysis was conducted according to the input seismic waves, so as to analyze the ground acceleration amplification characteristics. Ground acceleration amplification occurred as the ground rose above the reservoir embankments, and the maximum displacement was caused at the top of the embankment. In the top area, the ground acceleration amplification characteristics in the reservoir embankments offered up to 1.2 to 1.4-fold acceleration amplification compared to the bottom ground.

To define the behavioral characteristics of reservoir embankments, seismic-wave-charged dynamic centrifuge model tests were conducted to measure and analyze the earthquake response acceleration, displacement, and pore water pressure $[4,5]$. The acceleration amplification characteristics show that the amplification is large at the top. The lower the acceleration level, the larger the amplification ratio. The amplification of the acceleration at the top of the body was doubled, and the acceleration of the body was abruptly accelerated at 3/4 of the height of the dam. It can be seen that the settlement behavior of the body is large after the earthquake as the acceleration magnitude of the input seismic wave increases. The difference between the location of the lower slope and the top was not significant.

In this study, the seismic performance of agricultural reservoir embankments was evaluated and the seismic behavioral characteristics of agricultural reservoir embankments were analyzed by seismic wave to analyze the vulnerable areas of the facilities and to secure data for seismic design and reinforcement. The findings of this study can be used to conduct a safety diagnosis of agricultural infrastructures, and to perform an emergency investigation of the first-priority target area following the occurrence of earthquakes. 


\section{References}

[1] D. S. Kim, N. R. Kim, Y. W. Choo, G. C. Cho, "A newly developed state-of-the-art geotechnical centrifuge in Korea," KSCE Journal of Civil Engineering, vol. 17, no. 1, pp. 77-84, 2013.

[2] D. S. Kim, S. H. Lee, Y. W. Choo, "Self-balanced Earthquake Simulator on Centrifuge and Dynamic Performance Verification," KSCE Journal of Civil Engineering, vol. 17, no. 4, pp. 651-661, 2013.

[3] S. H. Lee, Y. W. Choo, Dong-Soo Kim, "Performance of an Equivalent Shear Beam (ESB) Model Container for Dynamic Geotechnical Centrifuge Tests," Soil Dynamics and Earthquake Engineering, vol. 44, pp. 102-114, 2013.

[4] C. W. Lee, Y. S. Kim, Y. S. Yoon, D. S. Chang, Y. W. Choo and M. C. Jung, "Stability Study of a Tide Embankment Subjected to Sea Level Variations Using Centrifugal Model Tests," Marine Georesources \& Geotechnology, vol. 33, no. 5, pp. 376-390, 2015.

[5] T. H. Kim and Y. S. Kim, "Amplification Characteristics at Artificial Island Considering Viscoelastic Effects of Cohesive Soil," Scientific Research and Essays, vol. 6, no. 4, pp. 809-818. 2011. 